Supporting Information for

\title{
Self-Assembly of Carbon Black/AAO Templates on Nanoporous Si for Broadband Infrared Absorption
}

Hui $\mathrm{Li}^{1}$, Liang $\mathrm{Wu}^{2}$, Heng Zhang ${ }^{1}$, Wei Dai ${ }^{1}$, Jiaming Hao ${ }^{4}, H a o \mathrm{Wu}^{3}$, *, Feng Ren ${ }^{2}$, and

$$
\text { Chang } \operatorname{Liu}^{1,} \text { * }
$$

${ }^{1}$ Key Laboratory of Artificial Micro- and Nano-structures of Ministry of Education, and School of Physics and Technology, Wuhan University, Wuhan 430072, China

${ }^{2}$ School of Physics and Technology, Center for Ion Beam Application and Hubei Nuclear Solid Physics Key Laboratory, Wuhan University, Wuhan 430072, China

${ }^{3}$ Hubei Nuclear Solid Physics Key Laboratory, and School of Physics and Technology, Wuhan University, Wuhan 430072, China

4 State Key Laboratory of Infrared Physics, Shanghai Institute of Technical Physics, Chinese Academy of Science, Shanghai 200083, China

* Correspondence: $\underline{\text { h.wu@whu.edu.cn and chang.liu@whu.edu.cn }}$ 


\section{S1. The transmissivity of heavily doped Si}

The transmission spectrum of heavily doped Si was measured by using a Fourier transform

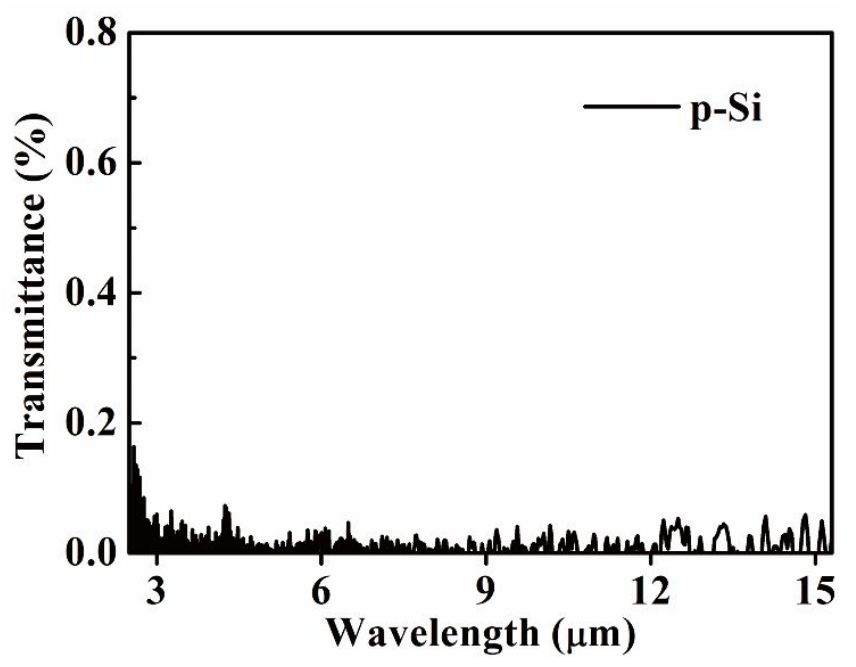

Figure S1. Transmission spectrum of heavily doped $\mathrm{Si}$

infrared (FTIR, Nicolet 6700) spectroscopy, as shown in Fig. S1. The result shows the transmittance is close to zero.

\section{S2. The reflectivity of heavily doped $\mathrm{Si}$}

(a)

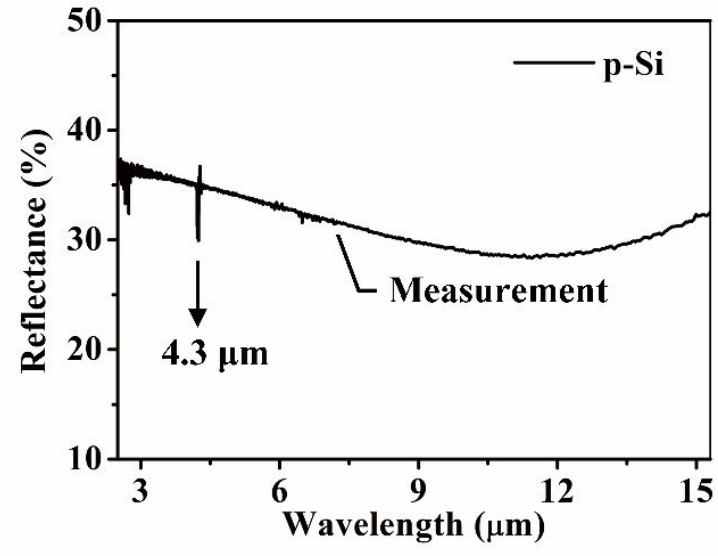

(b)

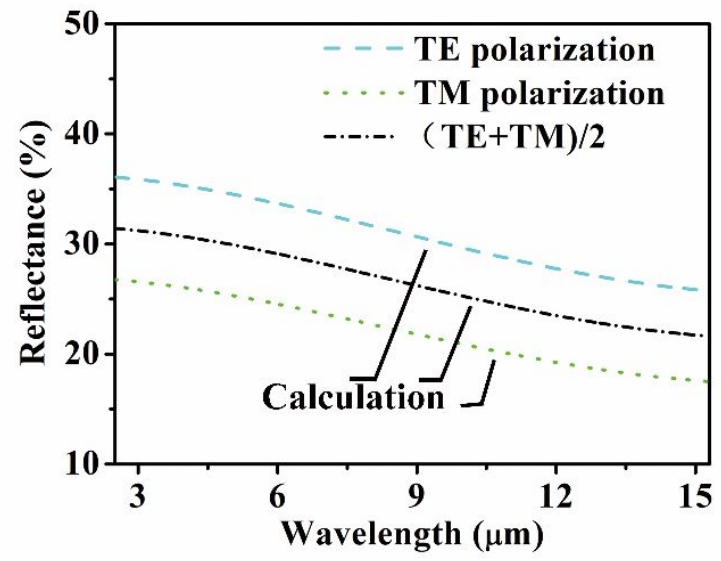

Figure S2. (a) Measured specular reflectance data of the single-crystalline Si. (b) Calculated wavelength-dependent reflectivities considering polarized (TE and TM) and unpolarized light (average of the TE and TM polarizations). 


\section{S3. The refractive index of heavily doped $\mathrm{Si}$}

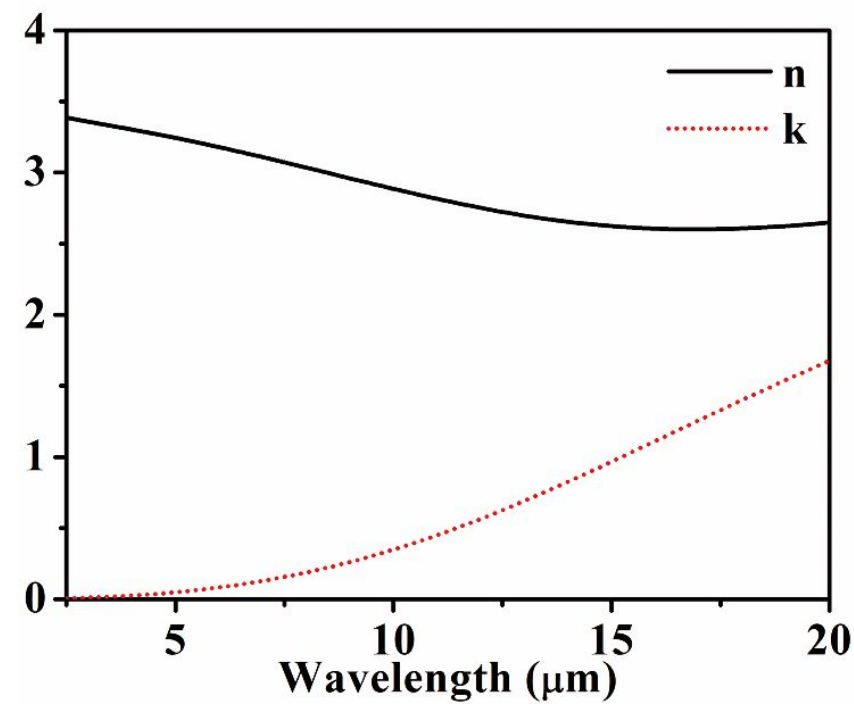

Figure S3. Complex refractive index (real and imaginary part) of

heavily doped Si

\section{S4. Multiple measurements of the structural reflectivity}

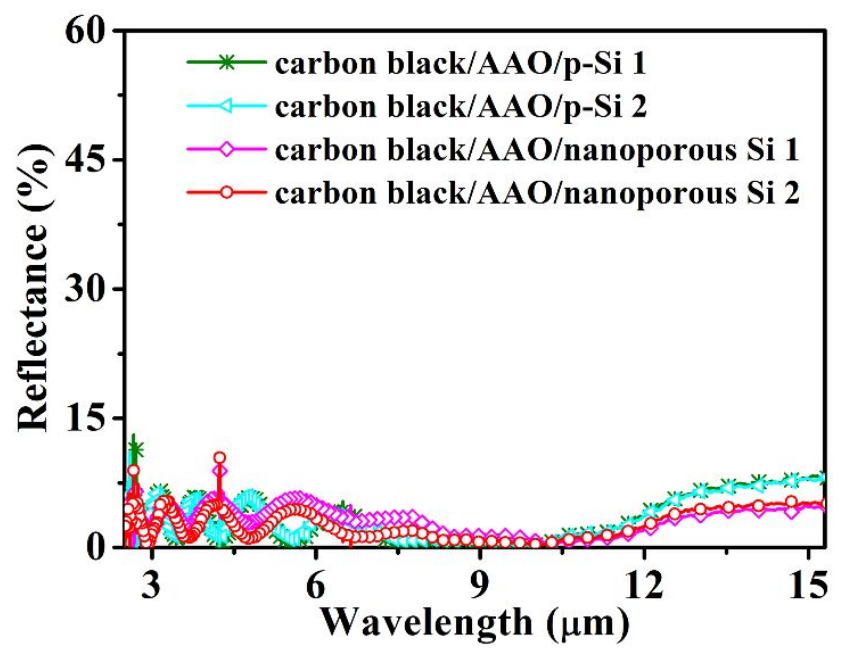

Figure S4. FTIR reflection spectra from two measurements.

\section{S5. The reflectivity of carbon black on single-crystalline $\mathrm{Si}$}

The amount of carbon black increases gradually on the surface of Si by extending the 
spraying time, as shown in Fig. S5(a-c). Particularly, when carbon black covers the surface of Si (green line), the reflectivity is reduced greatly compared to the Si. However, compared to the structures of carbon black onto AAO templates, the reflectivity of carbon black on Si is much higher, even increasing the amount of carbon black. In general, we emphasize that carbon black has a very strong infrared absorption, which is caused by extremely thick carbon black.
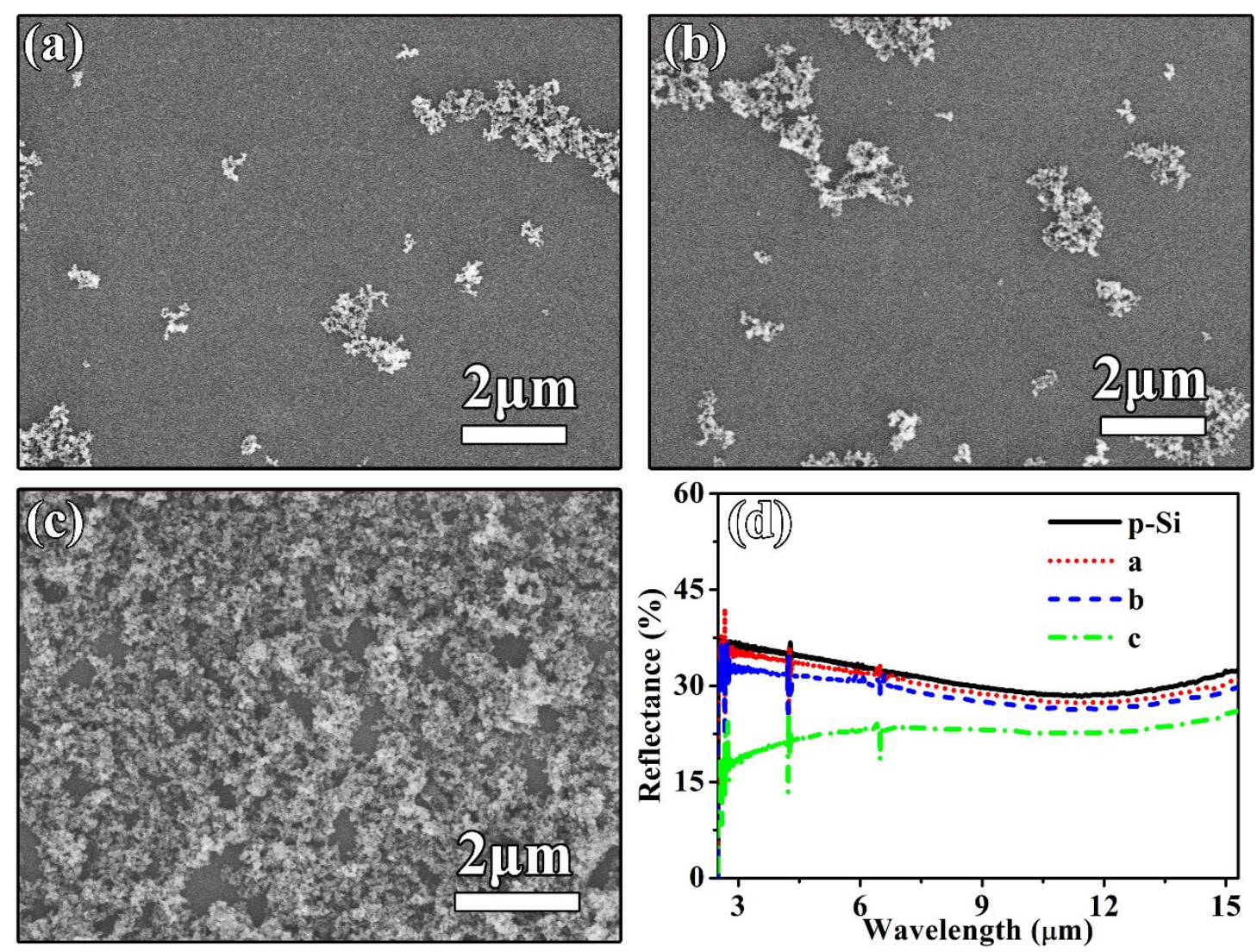

Figure S5. (a-c) Plane-view SEM image of different number of carbon black on single-crystalline Si. (d) FTIR measured reflection spectra. 


\section{S6. The effect of the number of carbon black on reflectivity}

We simulated the different number of carbon black particles onto AAO templates on heavily doped Si by FDTD. The reflection spectra are shown in Fig. S6. In the figure, the number 1 (red line) represents the total number of black carbon particles inside each pore is 858 in each pore. Accordingly, the number 2 (blue line) represents that total number is 2925 , and the number 3 (green line) represents that total number is 3900 . It is obvious that the reflectivity decreases gradually with the increase of carbon black particles in the range of $2.5-10.5 \mu \mathrm{m}$. However, the reflectivity increases slightly in the range of $\lambda>10.5 \mu \mathrm{m}$.

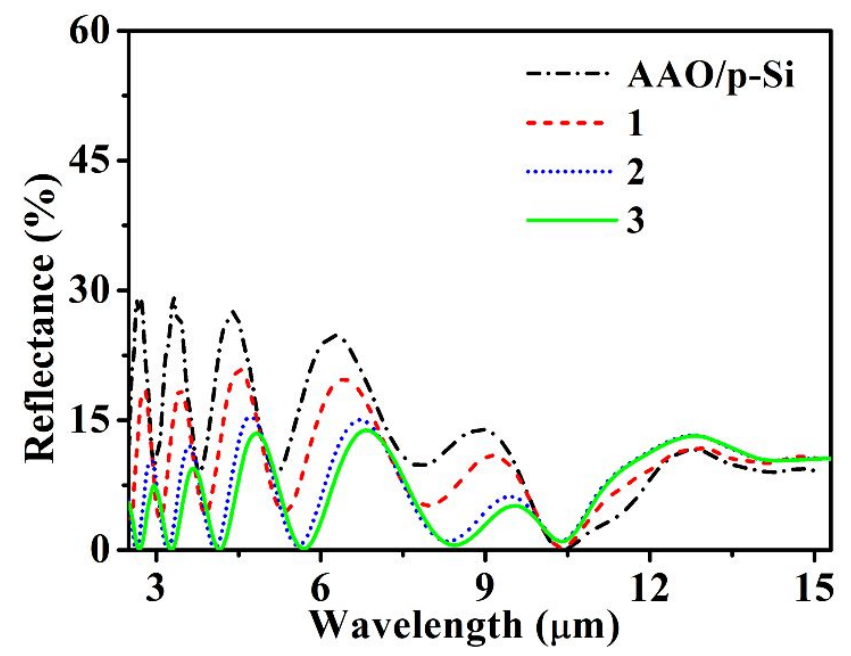

Figure S6. FDTD-calculated reflectivities of different number of carbon black particles onto AAO templates on $\mathrm{Si}$. 